\title{
Biochemical characterization of the enterotoxigenic Escherichia coli LeoA protein
}

\author{
Eric A. Brown and Philip R. Hardwidge \\ Center for Infectious Disease Research and Vaccinology, South Dakota State University, \\ Brookings, SD 57007, USA
}

Correspondence

Philip R. Hardwidge

hardwidg@gmail.com

Received 23 April 2007

Revised 13 July 2007

Accepted 26 July 2007

\begin{abstract}
Enterotoxigenic Escherichia coli (ETEC) causes enterotoxin-induced diarrhoea and significant mortality. The molecular mechanisms underlying how the heat-labile enterotoxin (LT) is secreted during infection are poorly understood. ETEC produce outer-membrane vesicles (OMVs) containing LT that are endocytosed into host cells. Although OMV production and protein content may be a regulated component of ETEC pathogenesis, how LT loading into OMVs is regulated is unknown. The LeoA protein plays a role in secreting LT from the bacterial periplasm. To begin to understand the function of LeoA and its role in ETEC $\mathrm{H} 10407$ pathogenesis, a site-directed mutant lacking the putative GTP-binding domain was constructed. The ability of wild-type and mutant LeoA to hydrolyse GTP in vitro was quantified. This domain was found to be responsible for GTP binding; it is important to LeoA's function in LT secretion, and may play a modest role in the formation and protein content of OMVs. Deletion of leoA reduced the abundance of OmpX in outer-membrane protein preparations and of LT in OMVs. Immunoprecipitation experiments revealed that LeoA interacts directly with OmpA, but that the GTP-binding domain is nonessential for this interaction. Deletion of leoA rendered ETEC H10407 non-motile, through apparent periplasmic accumulation of FliC.
\end{abstract}

\section{INTRODUCTION}

Enterotoxigenic Escherichia coli (ETEC) causes enterotoxin-induced diarrhoea and significant morbidity and mortality in humans and livestock (Berberov et al., 2004). ETEC was first characterized as the causative agent of travellers' diarrhoea in separate studies of military personnel in Aden (Rowe et al., 1970) and Vietnam (DuPont et al., 1971). The prototypical human strain, H10407 (O78:H11), expresses two different surface pili that serve as colonization factors (Cheney \& Boedeker, 1983). ETEC express several enterotoxins whose combined activities induce water and electrolyte loss from the intestine of infected subjects (Nataro \& Kaper, 1998). The heat-labile enterotoxin (LT) is structurally and functionally similar to the cholera toxin of Vibrio cholerae, inducing activation of adenylate cyclase in the host to alter normal electrolyte transport and cause diarrhoea (Spangler, 1992).

Despite its role as a major ETEC virulence determinant, only recently has significant effort been put into understanding how LT is secreted from the bacterium during infection (Tauschek et al., 2002). Earlier efforts failed to identify a protein secretory pathway, leading to the belief

Abbreviations: ETEC, enterotoxigenic Escherichia coli; LT, heat-labile enterotoxin; OMP, outer-membrane protein; OMV, outer-membrane vesicle; wt, wild-type. that ETEC release LT only during lysis (Wai et al., 1995). It was later realized that a type II secretion system, homologous to that used by $V$. cholerae to transport cholera toxin, is used to transport LT (Tauschek et al., 2002). As LT does not remain in the ETEC periplasm, but is instead found on the cell exterior (Horstman \& Kuehn, 2000), the involvement of outer-membrane vesicles (OMVs) in providing a point of display for LT was then recognized (Horstman \& Kuehn, 2002).

Gram-negative bacteria produce OMVs (Kesty et al., 2004), which in pathogenic strains are used to secrete virulence genes and proteins. Shiga toxins are delivered from E. coli O157:H7 into the host epithelium through OMVs (Kolling \& Matthews, 1999) and Helicobacter pylori releases the VacA toxin through OMV budding (Fiocca et al., 1999; Ilver et al., 2004). In addition to transporting virulence proteins, OMVs serve an important role in disseminating bacterial DNA. Pseudomonas aeruginosa OMV production increases in response to bacterial stress, and DNA isolated from OMVs can be expressed in other pathogenic isolates, implicating OMVs as an important mechanism of horizontal gene transfer (Kadurugamuwa \& Beveridge, 1997). LT is highly enriched in ETEC vesicles (Horstman \& Kuehn, 2000), whose endocytosis is dependent on cholesterol-rich lipid rafts found on the host epithelial surface (Kesty et al., 2004). OMVs derived from ETEC, but not from non-pathogenic E. coli strains, associate with host cells in a time- and receptor-dependent manner (Kesty 
et al., 2004), suggesting that OMV production and protein content may be a regulated component of ETEC pathogenesis. Release of OMVs also enhances survival during bacterial stress or during accumulation of misfolded proteins (McBroom \& Kuehn, 2007).

How the protein content of bacterial OMVs is regulated is unknown. Vesiculation may be a directed process as observed in eukaryotes, or, more likely, deformation of the bacterial envelope due to particular localization of the protein secretion machinery may focus secretion loci (Kesty et al., 2004). The leoA (labile enterotoxin output) gene was discovered in a mutational screen designed to find ETEC genes that confer the ability to invade intestinal cells in vitro. An in-frame deletion in leoA resulted in diminished LT secretion and a lack of fluid accumulation in a rabbit ileal loop model of infection, apparently due to a failure to secrete LT from the bacterial periplasm (Fleckenstein et al., 2000). E. coli possessing the pathogenicity island in which $l e o A$ is encoded secrete LT, while $E$. coli lacking the pathogenicity island retain LT in the periplasm (Fleckenstein et al., 2000). LeoA is homologous to proteins from bacterial secretion apparatuses, including EpsE from $V$. cholerae, which is required for cholera toxin secretion (Fleckenstein et al., 2000). In this study, we present data from experiments designed to characterize the biochemical activities of LeoA, the role of its GTP-binding domain in LT secretion, and its interaction with ETEC outer-membrane proteins.

\section{METHODS}

Construction and acquisition of $\Delta / e \circ A$ mutants. We acquired from Dr James Fleckenstein (University of Tennessee Health Science Center) an ETEC H10407 $\Delta$ leoA strain (Fleckenstein et al., 2000). A site-directed mutant was constructed through overlap PCR (Warrens et al., 1997), in which the putative GTP-binding site (GAFSDGKT) of LeoA was replaced with an inert sequence (GAGAGAGA). PRH-534, and PRH-557 and PRH-535 and PRH-558 (see Table 1 for oligonucleotide sequences), were combined in separate PCR reactions with ETEC H10407 genomic DNA. Products from these reactions were purified, combined in a third PCR, and amplified with PRH-534 and PRH-535 to generate a leoA amplicon containing the GTPbinding site substitution $(l e o A \Delta \mathrm{G})$. This PCR product was cloned into PCR2.1-TOPO and then subcloned into pFLAG-CTC for complementation studies and into pET28a for protein purification.

Protein purification. Standard molecular biology techniques were employed to subclone leoA into pET-28a. This construct was expressed in E. coli BL21(DE3). A $250 \mathrm{ml}$ culture was grown to an $\mathrm{OD}_{600}$ of 0.4 , when IPTG was added to $1 \mathrm{mM}$. After $3 \mathrm{~h}$ additional growth, cells were pelleted and resuspended in sonication buffer ( $5 \mathrm{mM}$ imidazole, $250 \mathrm{mM} \mathrm{NaCl}, 20 \mathrm{mM}$ Tris/HCl pH 7.9). Cells were sonicated and centrifuged to clarify the supernatant. The supernatant was added to packed pre-equilibrated Ni-NTA agarose and incubated for $1 \mathrm{~h}$ at $4{ }^{\circ} \mathrm{C}$. The slurry was poured into a disposable Poly-Prep chromatography column, washed five times with wash buffer $(60 \mathrm{mM}$ imidazole, $250 \mathrm{mM} \mathrm{NaCl}, 20 \mathrm{mM}$ Tris/ $\mathrm{HCl}$ $\mathrm{pH}$ 7.9). His-LeoA was eluted in $1 \mathrm{ml}$ fractions of wash buffer supplemented with increasing concentrations of imidazole and analysed by $10 \%$ SDS-PAGE. Fractions containing His-LeoA were dialysed into storage buffer $(20 \mathrm{mM}$ Tris/ $\mathrm{HCl}$ pH 7.0, $20 \mathrm{mM} \mathrm{NaCl}$, $1 \mathrm{mM}$ DTT, 5\% glycerol) using a Slide-A-Lyser dialysis cassette (Pierce). The His-LeoA $\Delta \mathrm{G}$ mutant was purified under denaturing (6 $\mathrm{M}$ guanidine. $\mathrm{HCl}$ ), but otherwise identical conditions, and refolded by stepwise dialysis into storage buffer.

Quantification of GTPase activity. The Enzchek Phosphate assay kit (Molecular Probes) was utilized according the manufacturer's specifications. A $1 \mu \mathrm{g}$ sample of purified His-LeoA or His-LeoA $\Delta \mathrm{G}$ was added to $1 \mathrm{ml}$ reaction mixtures preincubated for $10 \mathrm{~min}$ at $22{ }^{\circ} \mathrm{C}$ that contained $1 \times$ reaction buffer, MESG substrate and $1 \mathrm{U}$ purine nucleoside phosphorylase. Absorbance at $360 \mathrm{~nm}$ was monitored as a function of time.

Quantification of LT secretion. Subcultures from frozen bacterial stocks were grown overnight in CYE-glucose medium at $37^{\circ} \mathrm{C}$ with vigorous aeration and pelleted by centrifugation at $16000 \mathrm{~g}$ for $15 \mathrm{~min}$. The resulting clarified bacterial supernatants were frozen at $-80{ }^{\circ} \mathrm{C}$. To measure LT retained in the periplasm, bacterial pellets were washed twice in $1 \mathrm{ml}$ cold phosphate-buffered saline (PBS) and

Table 1. Oligonucleotides utilized in this study

Oligonucleotide name and purpose are indicated. Restriction sites used for cloning are designated in italics. The mutagenic primer region utilized in overlap PCR is shown in bold.

\begin{tabular}{|c|c|}
\hline Oligonucleotide & DNA sequence $\left(5^{\prime}-3^{\prime}\right)$ \\
\hline PRH534 BamHI leoA f & $\mathrm{G}_{2} \mathrm{C}_{2} \mathrm{GGATCCATG}_{2} \mathrm{~A}_{2} \mathrm{CA}_{2} \mathrm{~T}_{2} \mathrm{CA}_{3} \mathrm{CAGT}_{2} \mathrm{C}$ \\
\hline PRH535 XhoI leoA r & $\mathrm{G}_{2} \mathrm{C}_{2} \mathrm{CTCGAGCTATCTG}_{2}$ CAGTATG $_{2} \mathrm{~T}_{4} \mathrm{C}$ \\
\hline PRH538 XhoI leoA f & $\mathrm{G}_{2} \mathrm{C}_{2} \mathrm{CTCGAGATG}_{2} \mathrm{~A}_{2} \mathrm{CA}_{2} \mathrm{~T}_{2} \mathrm{CA}_{3} \mathrm{CAGT}_{2} \mathrm{C}$ \\
\hline PRH539 BglII leoA r & $\mathrm{G}_{2} \mathrm{C}_{2} \mathrm{AGATCTCTATCTG}_{2}$ CAGTATG $_{2} \mathrm{~T}_{4} \mathrm{C}$ \\
\hline PRH557 SOE leoA r & $\mathrm{C}_{2} \mathrm{ATGCG}_{2} \mathrm{CGATA}_{2} \mathrm{CGCT}_{2} \mathbf{C}_{2} \mathbf{A C}_{2} \mathbf{G C}_{2} \mathbf{T C}_{2} \mathbf{A C}_{2} \mathbf{T C}_{2} \mathbf{G C}_{5} \mathrm{~A}_{2} \mathrm{CAGAGCA}_{2} \mathrm{TACG}$ \\
\hline PRH558 SOE leoA f & $\mathrm{CGTAT}_{2} \mathrm{GCTCTGT}_{2} \mathrm{G}_{5} \mathbf{C G}_{2} \mathbf{A G}_{2} \mathbf{T G}_{2} \mathbf{A G}_{2} \mathbf{C G}_{2} \mathbf{T G}_{2} \mathbf{A}_{2} \mathbf{G C G T}_{2} \mathrm{ATCGC}_{2} \mathrm{GCATG}_{2}$ \\
\hline PRH633 EcoRI ompA f & $\mathrm{G}_{2} \mathrm{C}_{2} \mathrm{GAATTCATGA}_{5} \mathrm{GACAGC}$ \\
\hline PRH634 XhoI ompA r & $\mathrm{G}_{2} \mathrm{C}_{2} \mathrm{CTCGAGAGC}_{2} \mathrm{TGCG}_{2} \mathrm{CTGAG}$ \\
\hline PRH635 XhoI ompA f & $\mathrm{G}_{2} \mathrm{C}_{2} \mathrm{CTCGAGATGA}_{5}$ GACAGC \\
\hline PRH636 KpnI ompA r & $\mathrm{G}_{2} \mathrm{C}_{2} \mathrm{GGTACCAGC}_{2} \mathrm{TGCG}_{2} \mathrm{CTGAG}$ \\
\hline PRH637 EcoRI + 1 ompA f & $\mathrm{G}_{2} \mathrm{C}_{2}$ GAATTCCATGA ${ }_{5}$ GACAGC \\
\hline PRH638 EcoRI + 2 ompA $\mathrm{f}$ & $\mathrm{G}_{2} \mathrm{C}_{2}$ GAATTCTCATG $_{5}$ GACAGC \\
\hline
\end{tabular}


resuspended in an equal volume of buffer containing $25 \mathrm{mM}$ Tris/ $\mathrm{HCl}$ (pH 8.0), $10 \mathrm{mM}$ EDTA, lysozyme $\left(3 \mathrm{mg} \mathrm{ml}^{-1}\right)$, polymyxin B $\left(2 \mathrm{mg} \mathrm{ml}^{-1}\right)$, and phenylmethylsulfonyl fluoride $\left(8 \mu \mathrm{g} \mathrm{ml}^{-1}\right)$. After incubation on ice for $10 \mathrm{~min}$, cell pellets were subjected to repeated freeze-thaw cycles. After centrifugation $\left(16000 \mathrm{~g}, 15 \mathrm{~min}, 4{ }^{\circ} \mathrm{C}\right)$, clarified lysates were frozen at $-80{ }^{\circ} \mathrm{C}$ until used in LT assays. LT quantification was performed with a mixed-ganglioside ELISA (Horstman \& Kuehn, 2002).

Outer-membrane protein (OMP) isolation. OMPs were isolated as described by Achtman et al. (1983). Subcultures $(10 \mathrm{ml})$ from frozen bacterial stocks were grown overnight in $\mathrm{SOB}$ at $37{ }^{\circ} \mathrm{C}$ with vigorous aeration and pelleted by centrifugation $\left(16000 \mathrm{~g}, 15 \mathrm{~min}, 4^{\circ} \mathrm{C}\right)$. Bacterial pellets were washed twice in $1.5 \mathrm{ml} 10 \mathrm{mM} \mathrm{Tris} / \mathrm{HCl}$ $(\mathrm{pH} 8.0)$, resuspended a third time in this buffer, and lysed by sonication. Whole cells were removed by centrifugation $(10000 \mathrm{~g}$, $10 \mathrm{~min}, 4{ }^{\circ} \mathrm{C}$ ), the supernatants were aspirated into ultratubes, and Sarkosyl was added to $2 \%(\mathrm{w} / \mathrm{v})$. This mixture was incubated at room temperature for $30 \mathrm{~min}$ and then centrifuged $\left(38000 \mathrm{~g}, 1 \mathrm{~h}, 4{ }^{\circ} \mathrm{C}\right)$ to pellet the membrane proteins. The membrane protein pellet was washed with PBS, recentrifuged, and resuspended in sterile water. A $25 \mu \mathrm{g}$ sample of protein was mixed with SDS-loading buffer and separated by $10 \%$ SDS-PAGE.

Electron microscopy analysis of OMVs. OMVs were prepared by inoculating tryptic soy broth (TSB) with ETEC strains and incubating at $37{ }^{\circ} \mathrm{C}$ for $15 \mathrm{~h}$ with shaking (150 r.p.m.). Vesicles were harvested from the supernatant according to the method of Kesty et al. (2004). After incubation, cells were pelleted by centrifugation $(10000 \mathrm{~g}$, $10 \mathrm{~min}, 4^{\circ} \mathrm{C}$ ) and the supernatant was decanted and passed through a $0.22 \mu \mathrm{m}$-pore-size filter. Vesicles were collected by ultracentrifugation $\left(150000 \mathrm{~g}, 3 \mathrm{~h}, 4{ }^{\circ} \mathrm{C}\right)$, washed with PBS, resuspended in $100 \mu \mathrm{l}$ $\mathrm{mqH}_{2} \mathrm{O}$, and stored at $4{ }^{\circ} \mathrm{C}$. Samples $(10 \mu \mathrm{l})$ were placed on 300 mesh carbon-coated copper grids, allowed to settle on the film for $5 \mathrm{~min}$, then the excess was removed and the grids were stained for 5 min with $1 \%$ uranyl acetate. After drying, grids were viewed in a JEOL 1200EX transmission electron microscope and 10 random fields per grid were imaged for final vesicle counts. The protein content of OMVs was also analysed by $10 \%$ SDS-PAGE.

Immunoprecipitation of FLAG-LeoA. ETEC expressing FLAGLeoA, FLAG-LeoA $\Delta G$ or a FLAG epitope control were subcultured 1:50 into $10 \mathrm{ml} \mathrm{LB}$, grown for $3 \mathrm{~h}$ at $37^{\circ} \mathrm{C}$, and pelleted by centrifugation. After washing with PBS, bacteria were resuspended in PBS containing $1 \%$ DMSO and incubated for $30 \mathrm{~min}$ at room temperature. Saturated Tris was added to $100 \mathrm{mM}$ and incubation was continued for $10 \mathrm{~min}$. Cells were pelleted by centrifugation, resuspended in lysis buffer ( $1 \%$ Triton X-100, $10 \mathrm{mM}$ Tris/ $\mathrm{HCl}$, $\mathrm{pH} 7.0,1 \mathrm{mM}$ PMSF), and sonicated. Following centrifugation to remove insoluble material, the supernatant was incubated for $2 \mathrm{~h}$ at $4{ }^{\circ} \mathrm{C}$ with $5 \mu \mathrm{g}$ mouse-anti-FLAG antibody with gentle rotation. Then $25 \mu$ protein G-Sepharose beads (pre-blocked with $1 \%$ BSA) was added and the mixture was incubated overnight at $4{ }^{\circ} \mathrm{C}$ with gentle rotation. Beads were pelleted by centrifugation $\left(2000 \mathrm{~g}, 1 \mathrm{~min}, 4^{\circ} \mathrm{C}\right)$, washed three times with $1 \mathrm{ml}$ lysis buffer. The final wash was removed with a 25-gauge needle, the pellet was resuspended in SDS sample buffer, and samples were analysed by $10 \%$ SDS-PAGE.

Co-immunoprecipitation. BL21(DE3) cells were co-transformed with plasmids expressing FLAG- and His-tagged forms of LeoA, LeoA $\Delta \mathrm{G}$ and OmpA. Immunoprecipitations were conducted with protein G-Sepharose as described above. Mock immunoprecipitations of cultures expressing only a single construct were conducted to demonstrate antibody specificity.

Bacterial two-hybrid assay. ETEC $l e o A$, leoA $\triangle \mathrm{G}$ and ompA were sublconed into the BacterioMatch two-hybrid system vector kit bait
$(\mathrm{pBT})$ and target (pTRG) plasmids (Stratagene). Plasmids were cotransformed into XL1 Blue MRF [kanamycin-resistant $\left.\left(\operatorname{Kan}^{\mathrm{r}}\right)\right]$ and selected on LB agar plates containing the following antibiotics ( $\mu \mathrm{g}$ $\mathrm{ml}^{-1}$ ): kanamycin (50), chloramphenicol (25), tetracycline (10) and carbenicillin (0 to 500). Positive protein-protein interactions in the assay turn on the bla reporter gene, yielding a transformant resistant to carbenicillin $\left(\mathrm{Cb}^{\mathrm{r}}\right)$. Transformation controls were performed on plates lacking $\mathrm{Cb}$. Interaction controls were performed by cotransforming with empty $\mathrm{pBT}$ and $\mathrm{pTRG}$ vectors and with vectors expressing an unrelated fusion protein (E. coli O157:H7 EspG).

Periplasmic protein isolation and motility assays. ETEC strains were subcultured 1:50 into $5 \mathrm{ml} \mathrm{LB}$, grown for $3 \mathrm{~h}$ at $37^{\circ} \mathrm{C}$, and pelleted by centrifugation. The bacterial pellet was washed with $10 \mathrm{ml}$ PBS and the centrifugation was repeated. The supernatant was decanted and the pellet was resuspended in $1 \mathrm{ml} 50 \mathrm{mM}$ Tris pH 7.0, $20 \%$ sucrose, $10 \mathrm{mM}$ EDTA, $0.25 \mathrm{mg}$ lysozyme $\mathrm{ml}^{-1}$. The suspension was incubated at room temperature for $10 \mathrm{~min}$ and centrifuged at $8000 \mathrm{~g}, 10 \mathrm{~min}, 4{ }^{\circ} \mathrm{C}$. The supernatant was retained as the periplasmic fraction. Bacterial motility was evaluated by inoculating with a needle semisolid agar tubes containing BBL motility test medium (Beckton Dickinson), as described by the manufacturer.

Protein identification by LC-ESI-MS/MS. Bands excised from protein gels were digested in-gel with trypsin (Promega) at $37{ }^{\circ} \mathrm{C}$ overnight. The tryptic peptide solution was transferred to a microcentrifuge tube, extracted with $1 \%$ formic acid (SigmaAldrich), $2 \%$ acetonitrile (ACN; Fisher) in water, followed by an extraction with $50 \% \mathrm{ACN} /$ water. Both extracts were combined with the tryptic peptide solution, concentrated in a SpeedVac and suspended in $1 \%$ formic acid, $2 \%$ ACN. Peptide analysis was performed using LC-ESI-MS/MS. The peptides were desalted in-line and concentrated with an RP-Trap Symmetry300 C18 column, $5 \mu \mathrm{m}$ NanoEase (Waters), and separated using a C18 RP PepMap $75 \mu \mathrm{m}$ capillary column on a CapLC (Waters). Spectra were obtained in the positive-ion mode with a Q-TOF micro mass spectrometer (Micromass), deconvoluted, and analysed using the MassLynx software 4.0 (Micromass). Peptide matching and protein searches were performed using the ProteinLynx Global Server v 2.1 (Micromass) against the NCBInr v20060912 database.

\section{RESULTS AND DISCUSSION}

\section{LeoA is homologous to eubacterial and eukaryotic GTPases}

We performed a PSI-BLAST search to identify important functional domains and homologues of LeoA (Table 2). We identified a region of LeoA (sequence GAFSDGKT) corresponding exactly to the GTP-binding consensus sequence of $[\mathrm{AG}]-\mathrm{X}_{4}-\mathrm{G}-\mathrm{K}-[\mathrm{ST}]$. LeoA appears to contain an Era (E. coli Ras-like protein)-like domain, found in several essential bacterial GTPases involved in ribosome assembly (Inoue et al., 2003). We also detected significant homology between LeoA and an endoplasmic reticulumlocalized GTPase IMAP8 (immunity-associated protein 8) from eukaryotes. Significant homology was also observed to Sarlp (secretion-associated and Ras-related protein), a GTPase from Anabaena variabilis, a heterocyst-forming cyanobacterium. In eukaryotes, Sarl mediates the transport of proteins from the endoplasmic reticulum to the Golgi apparatus via the coat protein complex II [COPII (Miller et al., 2002)]. Sarl is believed to be an ancestral member of 
Table 2. PSI-BLAST identification of ETEC LeoA homologues

\begin{tabular}{|lllcc|}
\hline Protein & \multicolumn{1}{c}{ Organism } & \multicolumn{1}{c|}{ Annotation } & Identity (\%) & E value \\
\hline Cog1159 & Pseudomonas fluorescens & GTPase & 46 & $1 \times 10^{-157}$ \\
HP0731 & Helicobacter pylori & Predicted coding region & 30 & $1 \times 10^{-137}$ \\
JHP0668 & Helicobacter pylori & Hypothetical protein & 30 & $1 \times 10^{-135}$ \\
CE2856 & Corynebacterium efficiens & Hypothetical protein & 16 & $1 \times 10^{-103}$ \\
YP_154925 & Idiomarina loihiensis & Predicted GTPase & 15 & $1 \times 10^{-95}$ \\
Sar1p & Anabaena variabilis & GTPase Sar1 & 19 & $3 \times 10^{-77}$ \\
\hline
\end{tabular}

the Ras superfamily (Jekely, 2003) and is able to complement prokaryotic GTPases (Hartzell, 1997). While it is intriguing to speculate that the loading of protein cargo into bacterial OMVs may be in some way akin to GTPase-mediated control of protein sorting in eukaryotic secretory pathways, experiments from the Kuehn laboratory suggest that a 'cargo tag' is not required for protein sorting into bacterial vesicles (Kesty et al., 2004). Differential localization of type II secretion machinery may instead determine secretion loci (Kesty et al., 2004). Fleckenstein et al. (2000) also noted similarity to EpsE, the $V$. cholerae member of type II/type IV secretion NTPases that provide energy for bacterial protein secretion (Camberg \& Sandkvist, 2005). EpsE is known to have both a cytoplasmic (Abendroth et al., 2005) and innermembrane association (Camberg \& Sandkvist, 2005), dependent upon the expression of other Eps proteins.

\section{LeoA is a GTPase}

To quantify the ability of LeoA to hydrolyse GTP in vitro, we overexpressed and purified His-tagged forms of LeoA and a LeoA mutant (LeoA $\Delta \mathrm{G})$ in which the putative GTPbinding site (GAFSDGKT) was replaced, through overlap PCR (Warrens et al., 1997), with an inert sequence
(GAGAGAGA). These constructs were subcloned into pET28a (C-terminal His $_{6}$-tag) and expressed in E. coli BL21(DE3). We purified His-LeoA through Ni-NTA resin under native conditions (Fig. 1a). Because of reduced solubility in BL21(DE3) lysate, His-LeoA $\Delta \mathrm{G}$ was purified in the presence of $6 \mathrm{M}$ guanidine hydrochloride and refolded via stepwise dialysis. To serve as a refolding control in subsequent biochemical assays, we also purified His-LeoA under denaturing conditions.

To monitor in vitro GTP hydrolysis by His-LeoA and HisLeoA $\Delta \mathrm{G}, 1 \mu \mathrm{g}$ purified protein was added to the Enzchek Phosphate assay kit (Molecular Probes), which provides a sensitive GTPase assay system used previously to study the activity of a related family of GTPases (Zhuang et al., 2005). GTP hydrolysis was assayed every 15 s for 5 min by measuring the change in absorbance at $360 \mathrm{~nm}$ (Fig. 1b). For His-LeoA, $A_{360}$ changed linearly with respect to time (Fig. 1b; open triangles). However, addition of $1 \mu \mathrm{g}$ HisLeoA $\Delta \mathrm{G}$ did not result in efficient GTP hydrolysis, compared to His-LeoA (Fig. 1b; open squares). To exclude the possibility that this observation was due to the denaturing purification protocol employed for HisLeoA $\Delta \mathrm{G}$, we analysed the activity of His-LeoA purified under denaturing conditions (Fig. 1b; filled triangles). This protein behaved indistinguishably from native His-LeoA. (a)

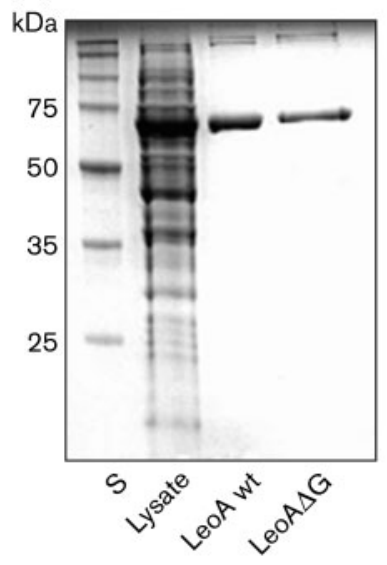

(b)

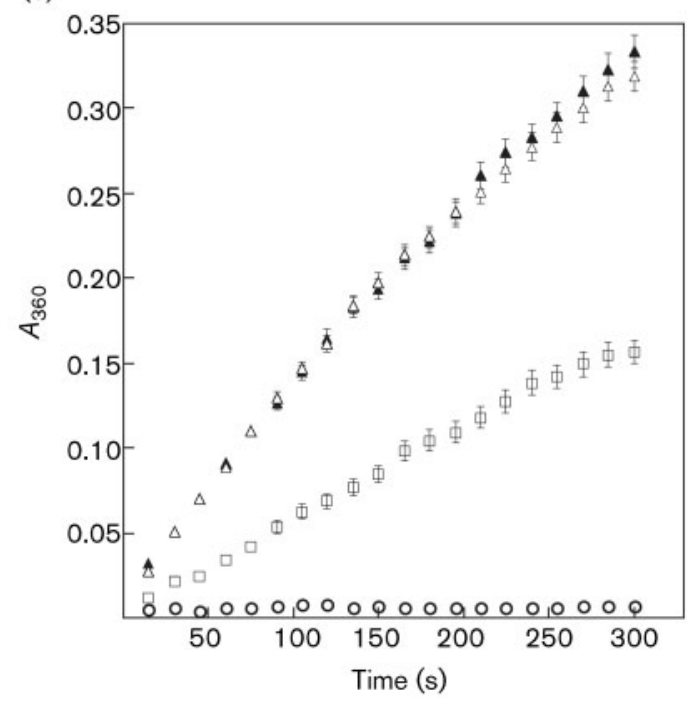

Fig. 1. LeoA is a GTPase. (a) Purification of His-LeoA. leoA was cloned into pET28a, expressed in E. coli BL21(DE3), purified by passage over pre-equilibrated Ni-NTA resin (Qiagen), and eluted with $300 \mathrm{mM}$ imidazole. Shown is a Coomassie-stained $10 \%$ SDSpolyacrylamide gel of the induced bacterial lysate, purified His-LeoA and His-Leo $\Delta$ G. S, Size standards. (b) Quantification of LeoA GTPase activity. GTP hydrolysis was monitored by measuring the change $A_{360}$ vs time, following the addition of $1 \mu \mathrm{g}$ LeoA proteins. The Enzchek Phosphate assay was performed according to the manufacturer's instructions (Invitrogen). Error bars (not shown if smaller than symbols) represent the standard deviation of three independent experiments. $\bigcirc$, Buffer; $\square$, His-Leo $\Delta G$; $\triangle$, His-LeoA native; $\boldsymbol{\Delta}$, HisLeoA refolded. 
This observation suggests that the deficient GTP hydrolysis observed with His-LeoA $\Delta \mathrm{G}$ is due solely to the sitedirected mutation, and not the purification method. Overall, these data suggest that LeoA is a functional GTPase.

\section{Deletion of IeoA alters OMP composition}

To determine the role of LeoA in maintaining OMP composition, we isolated OMPs from $\mathrm{H} 10407$ possessing or lacking LeoA and assessed changes in OMP composition by SDS-PAGE. We first complemented the $\Delta l e o A$ strain with plasmids expressing FLAG-tagged versions of LeoA (pleoA-FLAG) and leoA $\Delta \mathrm{G}$ (pleoA $\Delta \mathrm{G}-\mathrm{FLAG})$ and verified proper expression by Western blotting (Fig. 2a). OMPs were isolated and prepared by ultracentrifugation from lysates obtained from $10 \mathrm{ml}$ bacterial subcultures; $25 \mu \mathrm{g}$ of protein was analysed by $10 \%$ SDS-PAGE (Fig. 2b). Notably, a protein of $\sim 16 \mathrm{kDa}$ was present in greatly reduced abundance in the $\triangle l e o A$ strain, relative to wildtype (wt) H10407. Complementation with either LeoAFLAG or LeoA $\Delta$ G-FLAG restored protein abundance to wt levels. We excised this band and determined through mass spectrometry that this protein is OmpX.

OmpX is a member of a protein family that may be important to virulence by neutralizing host defences. Kuehn and co-workers noted in their pioneering studies of LT secretion via vesicles that OmpX is present in ETEC outer membrane and vesicle preparations, that the abundance of OmpX differs as a function of the growth medium, and that this protein is not detected in preparations from non-pathogenic strains (Horstman \& Kuehn, 2000). These authors also noted that OmpX is homologous to the Yersinia Ail protein, which has been suggested to promote bacterial invasion (Miller et al., 2001), and the OmpX adhesin of Enterobacter cloacae, which promotes invasiveness in rabbit ileal enterocytes (de Kort et al., 1994). It remains to be determined why deletion of LeoA alters OmpX abundance in the outer membrane.

\section{The GTPase domain of LeoA is essential to maximal LT secretion}

To quantify the role of the GTPase domain of LeoA in LT secretion, we measured the concentration of LT in the periplasmic and supernatant fractions of ETEC possessing or lacking leoA. We determined that in wt H10407, a significant amount of LT was secreted into culture supernatants (Table 3; $199.7 \pm 17.1 \mathrm{ng} \mathrm{ml}^{-1}$ ), whereas in the $\triangle l e o A$ strain, significantly less LT was detected $\left(34.5 \pm 4.3 \mathrm{ng} \mathrm{ml}^{-1}\right)$. Quantification of LT in the periplasmic fraction demonstrated a higher concentration in the $\triangle l e o A$ strain, in agreement with previous reports (Fleckenstein et al., 2000). When strains were complemented with plasmids expressing leoA, proper LT secretion was restored. However, in strains complemented with the pleoA $\triangle$ G-FLAG construct, LT secretion into culture supernatants did not return to wt levels. Surprisingly, wt ETEC transformed with pleoA $\Delta$ G-FLAG also displayed an LT secretion phenotype similar to the $\Delta l e o A$ strain. Overall, these data suggest that the GTPase domain of LeoA is required for maximal secretion of LT from the $\mathrm{H} 10407$ periplasm and suggest that expression of LeoA $\Delta \mathrm{G}$ may exert a dominant-negative phenotype.

To determine if introduction of LeoA would alter LT secretion in ETEC that naturally lack the leoA gene, we transformed pleoA-FLAG into two other $\mathrm{LT}^{+}$ETEC strains, 3030-2 $\left(\mathrm{K} 88^{+} \mathrm{STa}^{+} \mathrm{STb}^{+} \mathrm{LT}^{+}\right)$, a porcine field (a)
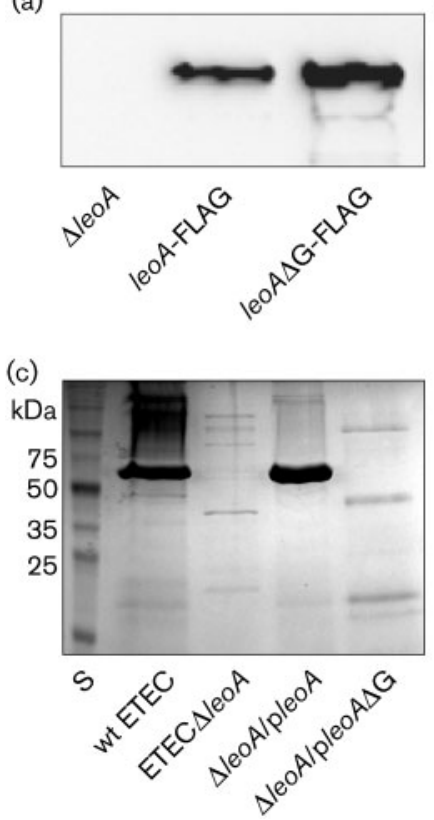

(b)

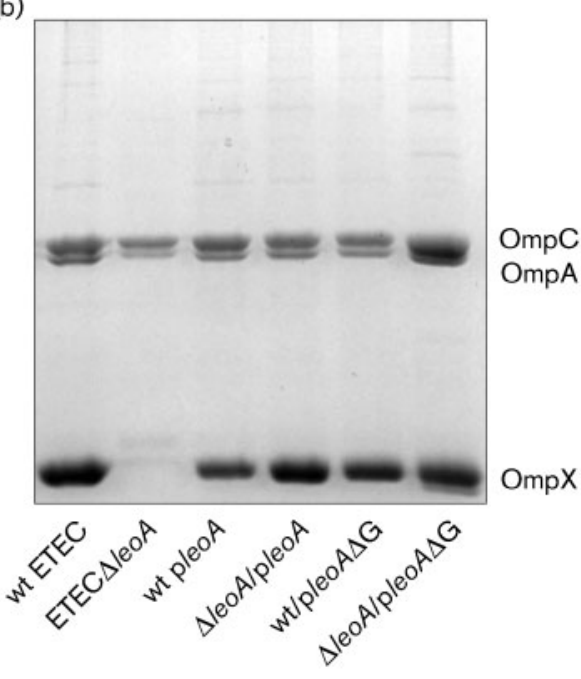

Fig. 2. Analysis of OMPs in ETEC strains either possessing or lacking LeoA. (a) Complementation of ETEC $\Delta / e o A$ with pleoAFLAG and pleoA $\triangle \mathrm{G}-\mathrm{FLAG}$. Bacterial lysates from the parent and complemented ETEC $\Delta / e o A$ strains were interrogated for the presence of FLAG-tagged LeoA and LeoA $\Delta G$ by Western blotting using a mouse anti-FLAG antibody. (b) OMP composition. OMPs from the indicated strains were isolated and subjected to $10 \%$ SDS-PAGE. Annotated bands were excised from gels and identified by mass spectrometry. (c) OMV composition. Protein content of OMVs isolated from the indicated strains was assessed by Coomassie G-250 staining of samples separated by $10 \%$ SDSPAGE. S, size standards. 
Table 3. Quantification of LT secretion in ETEC strains possessing or lacking leo $A$

\begin{tabular}{|c|c|c|}
\hline \multirow[t]{2}{*}{ Strain } & \multicolumn{2}{|c|}{ LT $\left(\mathrm{ng} \mathrm{ml}^{-1}\right)$} \\
\hline & Periplasmic & Supernatant \\
\hline wt ETEC & $15.2 \pm 4.5$ & $199.7 \pm 17.1$ \\
\hline ETEC $\Delta l e o A$ & $27.0 \pm 7.0^{*}$ & $34.5 \pm 4.3 \dagger$ \\
\hline wt ETEC/pleoA-FLAG & $32.3 \pm 9.2^{*}$ & $124.7 \pm 8.2 \dagger$ \\
\hline ETEC $\Delta l e o A / p l e o A-F L A G$ & $24.8 \pm 12.0^{*}$ & $126.3 \pm 10.1 \dagger$ \\
\hline wt ETEC/pleoA $\Delta \mathrm{G}$-FLAG & $20.5 \pm 8.4$ & $32.7 \pm 5.7 \dagger$ \\
\hline ETEC $\Delta l e o A / p l e o A \Delta \mathrm{G}-\mathrm{FLAG}$ & $7.2 \pm 4.4^{\star}$ & $31.2 \pm 6.4 \dagger$ \\
\hline
\end{tabular}

${ }^{\star}$ Significantly different from wt; Student's $t$-test, $P<0.05$.

$\dagger$ Significantly different from wt; Student's $t$-test, $P<0.005$.

isolate (Erickson et al., 1992), and 91.1283 (O6:STa ${ }^{+}$ $\mathrm{LT}^{+}$), a pathogenic human isolate (Chobi DebRoy, personal communication). Whereas expression of LeoA in 3030-2 increased LT secretion by $\sim 14 \%$, transformation of 91.1283 caused no detectable change.

\section{LeoA is important to the formation of OMVs}

To determine the role of LeoA in the formation of OMVs, we purified OMVs from ETEC either possessing or lacking leoA by ultracentrifugation and filtration (Kadurugamuwa \& Beveridge, 1997) and quantified by electron microscopy the number of OMVs produced by each strain. Vesicle preparations from wt $\mathrm{H} 10407$ contained $15.2 \pm 3.2$ OMVs per grid, whereas $\Delta l e o A$ produced $4.0 \pm 1.8$. Complementation of $\triangle l e o A$ with pleoA-FLAG partially restored the production of OMVs $(8.0 \pm 3.4)$, whereas complementation with pleoA $\Delta \mathrm{G}-\mathrm{FLAG}$ actually reduced the number of OMVs observed $(1.4 \pm 3.1)$. Although gross morphological differences were not observed among OMVs produced by wt vs $\Delta l e o A$, it is possible that these OMVs may differ in their affinity for the electron microscopy grid.

We also sought to determine the extent to which OMV protein content differed among complemented strains by subjecting purified OMVs to SDS-PAGE. As shown in Fig. 2(c), the total protein content and distribution of specific proteins within OMVs differed substantially between wt and $\triangle l e o A$. Whereas complementation with pleoA-FLAG restored the wt OMV protein profile, complementation with pleoA $\Delta$ G-FLAG did not.

We also quantified the extent to which LT could be detected in OMVs purified from ETEC possessing or lacking LeoA. OMV-associated LT was found in wt H10407 $\left(0.15 \mathrm{ng} \mathrm{ml}^{-1}\right)$, although at a lower concentration than reported from ETEC 2 (Horstman \& Kuehn, 2000). We observed a slight reduction in LT concentration in OMVs isolated from $\triangle l e o A$ and $\triangle l e o A /$ pleoA $\Delta$ G-FLAG $(0.08$ and $0.07 \mathrm{ng} \mathrm{ml}^{-1}$, respectively), but not from $\Delta l e o A / \mathrm{pleoA}$ FLAG $\left(0.13 \mathrm{ng} \mathrm{ml}^{-1}\right)$. These data suggest that LeoA may play a modest role in OMV formation and that the GTPbinding domain is important to this process.

\section{LeoA interacts with OmpA}

To identify ETEC proteins that interact with LeoA, we expressed FLAG-tagged versions of LeoA, LeoA $\Delta \mathrm{G}$ and a FLAG-epitope control in ETEC H10407. ETEC lysates were incubated with an anti-FLAG antibody, immunoprecipitated with protein G Sepharose, and analysed by SDSPAGE. As shown in Fig. 3(a), LeoA could be immunoprecipitated from both the pleoA-FLAG and the pleoA $\Delta \mathrm{G}-$ FLAG strains (top asterisk). An additional protein band immunoprecipitated from both the pleoA-FLAG and the pleoA $\triangle$ G-FLAG samples, but not in the FLAG-epitope control sample (bottom asterisk), was excised and identified by mass spectrometry as the outer-membrane protein OmpA.

OmpA is a major E. coli OMP with an important role in structural integrity and is believed to link physically the outer membrane with the peptidoglycan layer (Koebnik et al., 2000). In E. coli $\mathrm{K} 1$, OmpA is associated with neonatal meningitis through invasion of endothelial cells (Prasadarao et al., 1996). In E. coli O157: H7, OmpA plays a role in adherence to intestinal epithelial cells (Torres \& Kaper, 2003) and is also believed to mediate stimulation of dendritic cells to produce cytokines (Torres et al., 2006). Kuehn and co-workers demonstrated that ETEC OmpA is an outer-membrane component of native vesicles (Kesty et al., 2004). They observed that ETEC lacking LeoA fail to grow in a deoxycholate resistance assay, suggesting a loss of membrane integrity (Kesty et al., 2004). We observed that the $\Delta l e o A$ strain also has a significantly reduced growth rate in rich media (data not shown). An interaction between LeoA and OmpA could account for these observations.

To begin to confirm the putative interaction between LeoA and OmpA, ompA was cloned into pET28a and pFLAGCTC and coexpressed with His- and FLAG-tagged LeoA and LeoA $\Delta \mathrm{G}$ in BL21(DE3) cells. Lysates were subjected to immunoprecipitation with an anti-His antibody and interrogated by anti-FLAG immunoblotting. FLAGOmpA could only be immunoprecipitated by $\alpha$-His antibodies when coincubated with either His-LeoA or His-LeoA $\Delta G$ (Fig. 3b, left). The converse experiment, in which anti-FLAG antibody was used to immunoprecipitate OmpA, also resulted in the enrichment of both His-LeoA and His-LeoA $\Delta \mathrm{G}$ (Fig. 3b, right). Mock immunoprecipitations demonstrated that His-LeoA was not precipitated with anti-FLAG antibodies unless FLAG-OmpA was present. Similarly, FLAG-OmpA was not precipitated with anti-His antibodies in the absence of His-LeoA. Overall, these data support the notion that LeoA interacts with OmpA.

We also studied the interaction between LeoA and OmpA in a bacterial two-hybrid assay system. In this assay, plasmids expressing fusion proteins to a DNA-binding 
(a)

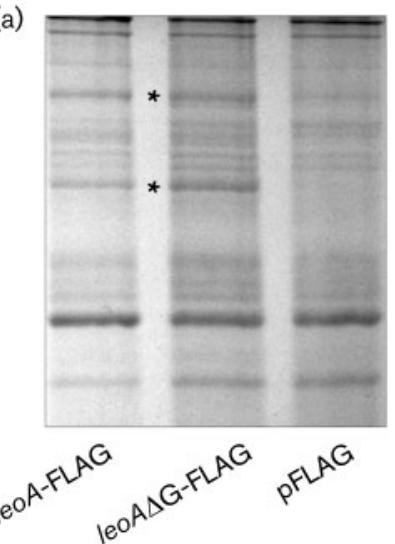

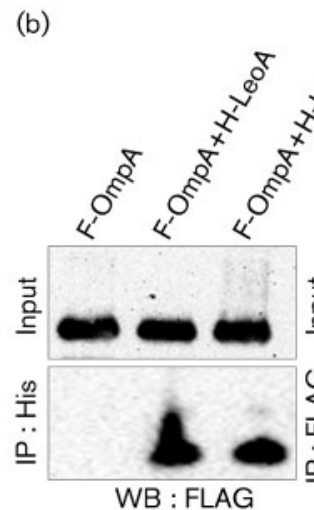
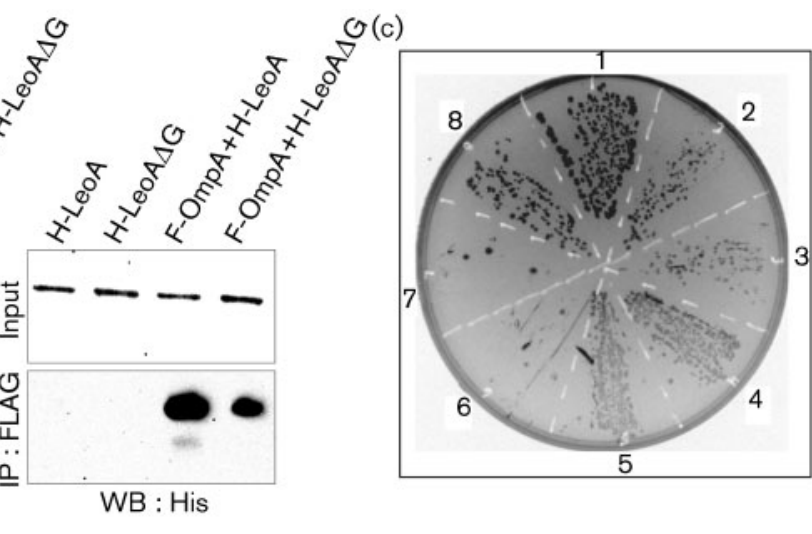

Fig. 3. LeoA interacts with OmpA. (a) Immunoprecipitation of FLAG-tagged LeoA from ETEC H10407. FLAG-tagged LeoA was immunoprecipitated from ETEC lysates and subjected to $10 \%$ SDS-PAGE. Bands selectively immunoprecipitated (indicated by asterisks) were identified by mass spectrometry as LeoA (upper) and OmpA (lower). (b) Co-immunoprecipitation experiments. His $(\mathrm{H})$ - and FLAG(F)-tagged forms of LeoA, LeoA $\triangle \mathrm{G}$ and OmpA were coexpressed in BL21(DE3) cells. Bacterial lysates were immunoprecipitated with either anti-His or anti-FLAG antibodies and interrogated by immunoblotting. Shown are the input and immunoprecipitated samples from experiments performed with both anti-His (left) and anti-FLAG (right) antibodies. (c) Bacterial two-hybrid analysis of interactions between LeoA and OmpA. Co-transformants harbouring the indicated plasmids were plated on selective medium $\left(\mathrm{LB}+300 \mu \mathrm{g} \mathrm{Cb} \mathrm{ml}{ }^{-1}\right)$ in the indicated plate octants: 1 , sepD-pBT sepLpTRG; 2, ompA-pBT leoA-pTRG; 3, ompA-pBT leoA $\Delta$ G-pTRG; 4, ompA-pTRG leoA-pBT; 5, ompA-pTRG leoA $\Delta$ G-pBT; 6 , leoA-pBT leoA-pTRG; 7, leoA $\Delta$ G-pBT leoA $\Delta$ G-pTRG; 8, ompA-pBT ompA-pTRG.

domain and a transcriptional activation domain are cotransformed into reporter cells. Protein-protein interaction strength is assessed semiquantitatively by growth on selective media. Co-transformation of either LeoA or LeoA $\Delta \mathrm{G}$ with OmpA conferred survival on selective medium (Fig. 3c, octants 2-5). The interaction between enteropathogenic E. coli E2348/69 SepD-SepL was monitored as a positive control [Fig. 3c, octant 1; (Deng et al., 2005)]. Reporter gene activation was also seen in cells that coexpressed reporter fusions to OmpA (Fig. 3c, octant 8). No growth was obtained in cells expressing only a single protein fusion, or with coexpression of LeoA constructs. No difference was observed in bacterial growth properties among strains transformed with LeoA or LeoA $\Delta \mathrm{G}$. We conclude from these data that the GTP-binding domain of LeoA appears nonessential in mediating binding to OmpA.

\section{Deletion of leoA affects H10407 motility}

We also interrogated the bacterial periplasm for evidence of proteins whose distribution might be affected by the presence or absence of LeoA. Significantly, a specific protein was highly increased in abundance in the periplasm of $\Delta l e o A$, relative to wt and the complemented strain $(\Delta \mathrm{leoA} /$ pleoA-FLAG; Fig. 4a, asterisk). We identified this protein by mass spectrometry as FliC. FliC is the major component of the bacterial flagellum (Aldridge et al., 2006), which has a proven role in E. coli motility (Gomez-Gomez et al., 2007), (a)

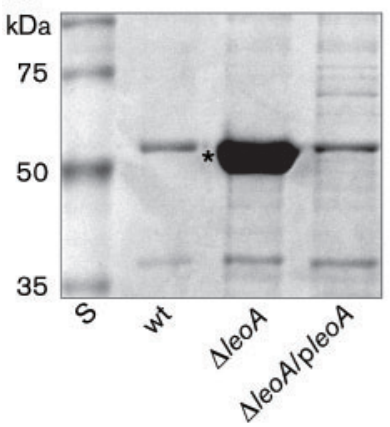

(b)

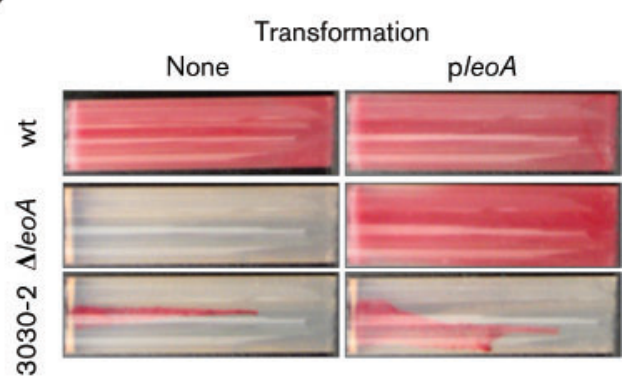

Fig. 4. LeoA deletion results in periplasmic accumulation of $\mathrm{FliC}$ and reduces bacterial motility. (a) Analysis of periplasmic proteins in ETEC strains either possessing or lacking LeoA. Periplasmic proteins from the indicated strains were isolated and subjected to $10 \%$ SDS-PAGE. The band indicated with an asterisk was excised and identified by mass spectrometry. (b) Bacterial motility assays. The indicated ETEC strains were inoculated into semisolid agar containing BBL motility test medium. Motility was evaluated $24 \mathrm{~h}$ postinoculation. 
host cell adherence (Giron et al., 2002; Wright et al., 2005), invasion (Parthasarathy et al., 2007) and virulence (Giron et al., 2002; Parthasarathy et al., 2007).

While we did not observe significant differences in the adherence of wt and $\Delta l e o A$ H10407 to Caco-2 cells (data not shown), when we evaluated bacterial motility, $\Delta l e o A$ was significantly inhibited in its ability to swim through semisolid agar and complementation with pleoA-FLAG restored motility (Fig. 4b). Heterologous expression of LeoA in the porcine isolate 3030-2 modestly increased motility. Thus, in addition to roles in LT secretion and modulation of OMP composition, LeoA may in some way contribute to the formation of motility structures. However, it is unlikely that LeoA is generally important to ETEC motility, as the vast majority of characterized isolates are $l e o A^{-}$(see below; Turner et al., 2006). Our observations may instead result from the apparent pleiotropic nature of the leoA mutation on H10407 proteins associated with the periplasm or bacterial membranes.

It is interesting to speculate as to why LeoA appears to play a significant role in properties typically associated with H10407 virulence. The findings described here are of note because leoA is not typically found in ETEC isolates characterized to date. Turner et al. (2006) analysed the distribution of leoA by multilocus sequence typing of 209 different ETEC isolates and found that leoA was present in only $3 \%$ of the tested strains. These authors speculate that because numerous isolates possessing LT do not encode leo $A$, this gene is not generally required for ETEC pathogenicity (Turner et al., 2006). Ongoing and future experiments may elucidate how LeoA might contribute to the disease severity associated with H10407 and whether similar functions might be imparted by related virulence factors in isolates that have been less well characterized.

\section{ACKNOWLEDGEMENTS}

We thank James Fleckenstein (University of Tennessee Health Science Center) for valuable advice and for generously providing the $\Delta l e o \mathrm{~A}$ strain, Chobi DebRoy for providing ETEC 91.1283, Michael Chaussee, Eduardo Callegari (University of South Dakota; NIH Grant P20 RR016479 from the INBRE Program of the National Center for Research Resources) and Leonard Foster (University of British Columbia) for assistance with mass spectrometry, and Randy Tindall and Martin Katz of the Electron Microscopy Core Facility at the University of Missouri, Columbia. This work was conducted in part using the South Dakota State University Functional Genomics Core Facility and was supported in part by the National Science Foundation/ EPSCoR Grant 0091948 and by the State of South Dakota.

\section{REFERENCES}

Abendroth, J., Murphy, P., Sandkvist, M., Bagdasarian, M. \& Hol, W. G. (2005). The X-ray structure of the type II secretion system complex formed by the N-terminal domain of EpsE and the cytoplasmic domain of EpsL of Vibrio cholerae. J Mol Biol 348, 845-855.
Achtman, M., Mercer, A., Kusecek, B., Pohl, A., Heuzenroeder, M., Aaronson, W., Sutton, A. \& Silver, R. P. (1983). Six widespread bacterial clones among Escherichia coli K1 isolates. Infect Immun 39, 315-335.

Aldridge, P. D., Karlinsey, J. E., Aldridge, C., Birchall, C., Thompson, D., Yagasaki, J. \& Hughes, K. T. (2006). The flagellar-specific transcription factor, sigma28, is the type III secretion chaperone for the flagellarspecific anti-sigma28 factor FlgM. Genes Dev 20, 2315-2326.

Berberov, E. M., Zhou, Y., Francis, D. H., Scott, M. A., Kachman, S. D. \& Moxley, R. A. (2004). Relative importance of heat-labile enterotoxin in the causation of severe diarrheal disease in the gnotobiotic piglet model by a strain of enterotoxigenic Escherichia coli that produces multiple enterotoxins. Infect Immun 72, 3914-3924.

Camberg, J. L. \& Sandkvist, M. (2005). Molecular analysis of the Vibrio cholerae type II secretion ATPase EpsE. J Bacteriol 187, 249-256.

Cheney, C. P. \& Boedeker, E. C. (1983). Adherence of an enterotoxigenic Escherichia coli strain, serotype O78:H11, to purified human intestinal brush borders. Infect Immun 39, 1280-1284.

de Kort, G., Bolton, A., Martin, G., Stephen, J. \& van de Klundert, J. A. (1994). Invasion of rabbit ileal tissue by Enterobacter cloacae varies with the concentration of OmpX in the outer membrane. Infect Immun 62, 4722-4726.

Deng, W., Li, Y., Hardwidge, P. R., Frey, E. A., Pfeutzner, R. A., Lee, S., Gruenheid, S., Strynakda, N. C., Puente, J. L. \& Finlay, B. B. (2005). Regulation of type III secretion hierarchy of translocators and effectors in attaching and effacing bacterial pathogens. Infect Immun 73, 2135-2146.

DuPont, H. L., Formal, S. B., Hornick, R. B., Snyder, M. J., Libonati, J. P., Sheahan, D. G., LaBrec, E. H. \& Kalas, J. P. (1971). Pathogenesis of Escherichia coli diarrhea. $N$ Engl J Med 285, 1-9.

Erickson, A. K., Willgohs, J. A., McFarland, S. Y., Benfield, D. A. \& Francis, D. H. (1992). Identification of two porcine brush border glycoproteins that bind the K88ac adhesin of Escherichia coli and correlation of these glycoproteins with the adhesive phenotype. Infect Immun 60, 983-988.

Fiocca, R., Necchi, V., Sommi, P., Ricci, V., Telford, J., Cover, T. L. \& Solcia, E. (1999). Release of Helicobacter pylori vacuolating cytotoxin by both a specific secretion pathway and budding of outer membrane vesicles. Uptake of released toxin and vesicles by gastric epithelium. J Pathol 188, 220-226.

Fleckenstein, J. M., Lindler, L. E., Elsinghorst, E. A. \& Dale, J. B. (2000). Identification of a gene within a pathogenicity island of enterotoxigenic Escherichia coli $\mathrm{H} 10407$ required for maximal secretion of the heat-labile enterotoxin. Infect Immun 68, 2766-2774.

Giron, J. A., Torres, A. G., Freer, E. \& Kaper, J. B. (2002). The flagella of enteropathogenic Escherichia coli mediate adherence to epithelial cells. Mol Microbiol 44, 361-379.

Gomez-Gomez, J. M., Manfredi, C., Alonso, J. C. \& Blazquez, J. (2007). A novel role for RecA under non-stress: promotion of swarming motility in Escherichia coli K-12. BMC Biol 5, 14.

Hartzell, P. L. (1997). Complementation of sporulation and motility defects in a prokaryote by a eukaryotic GTPase. Proc Natl Acad Sci U S A 94, 9881-9886.

Horstman, A. L. \& Kuehn, M. J. (2000). Enterotoxigenic Escherichia coli secretes active heat-labile enterotoxin via outer membrane vesicles. J Biol Chem 275, 12489-12496.

Horstman, A. L. \& Kuehn, M. J. (2002). Bacterial surface association of heat-labile enterotoxin through lipopolysaccharide after secretion via the general secretory pathway. J Biol Chem 277, 32538-32545.

Ilver, D., Barone, S., Mercati, D., Lupetti, P. \& Telford, J. L. (2004). Helicobacter pylori toxin VacA is transferred to host cells via a novel contact-dependent mechanism. Cell Microbiol 6, 167-174. 
Inoue, K., Alsina, J., Chen, J. \& Inouye, M. (2003). Suppression of defective ribosome assembly in a $r b f A$ deletion mutant by overexpression of Era, an essential GTPase in Escherichia coli. Mol Microbiol 48, 1005-1016.

Jekely, G. (2003). Small GTPases and the evolution of the eukaryotic cell. Bioessays 25, 1129-1138.

Kadurugamuwa, J. L. \& Beveridge, T. J. (1997). Natural release of virulence factors in membrane vesicles by Pseudomonas aeruginosa and the effect of aminoglycoside antibiotics on their release. J Antimicrob Chemother 40, 615-621.

Kesty, N. C., Mason, K. M., Reedy, M., Miller, S. E. \& Kuehn, M. J. (2004). Enterotoxigenic Escherichia coli vesicles target toxin delivery into mammalian cells. EMBO J 23, 4538-4549.

Koebnik, R., Locher, K. P. \& Van Gelder, P. (2000). Structure and function of bacterial outer membrane proteins: barrels in a nutshell. Mol Microbiol 37, 239-253.

Kolling, G. L. \& Matthews, K. R. (1999). Export of virulence genes and Shiga toxin by membrane vesicles of Escherichia coli O157: H7. Appl Environ Microbiol 65, 1843-1848.

McBroom, A. J. \& Kuehn, M. J. (2007). Release of outer membrane vesicles by Gram-negative bacteria is a novel envelope stress response. Mol Microbiol 63, 545-558.

Miller, V. L., Beer, K. B., Heusipp, G., Young, B. M. \& Wachtel, M. R. (2001). Identification of regions of Ail required for the invasion and serum resistance phenotypes. Mol Microbiol 41, 1053-1062.

Miller, E., Antonny, B., Hamamoto, S. \& Schekman, R. (2002). Cargo selection into COPII vesicles is driven by the Sec24p subunit. EMBO J 21, 6105-6113.

Nataro, J. P. \& Kaper, J. B. (1998). Diarrheagenic Escherichia coli. Clin Microbiol Rev 11, 142-201.

Parthasarathy, G., Yao, Y. \& Kim, K. S. (2007). Flagella promote Escherichia coli $\mathrm{K} 1$ association with and invasion of human brain microvascular endothelial cells (HBMEC). Infect Immun 75, 2937-2945.

Prasadarao, N. V., Wass, C. A., Weiser, J. N., Stins, M. F., Huang, S. H. \& Kim, K. S. (1996). Outer membrane protein A of Escherichia coli contributes to invasion of brain microvascular endothelial cells. Infect Immun 64, 146-153.

Rowe, B., Taylor, J. \& Bettelheim, K. A. (1970). An investigation of traveller's diarrhoea. Lancet 1, 1-5.

Spangler, B. D. (1992). Structure and function of cholera toxin and the related Escherichia coli heat-labile enterotoxin. Microbiol Rev 56, 622-647.

Tauschek, M., Gorrell, R. J., Strugnell, R. A. \& Robins-Browne, R. M. (2002). Identification of a protein secretory pathway for the secretion of heat-labile enterotoxin by an enterotoxigenic strain of Escherichia coli. Proc Natl Acad Sci U S A 99, 7066-7071.

Torres, A. G. \& Kaper, J. B. (2003). Multiple elements controlling adherence of enterohemorrhagic Escherichia coli O157:H7 to HeLa cells. Infect Immun 71, 4985-4995.

Torres, A. G., Li, Y., Tutt, C. B., Xin, L., Eaves-Pyles, T. \& Soong, L. (2006). Outer membrane protein A of Escherichia coli O157:H7 stimulates dendritic cell activation. Infect Immun 74, 2676-2685.

Turner, S. M., Chaudhuri, R. R., Jiang, Z. D., DuPont, H., Gyles, C., Penn, C. W., Pallen, M. J. \& Henderson, I. R. (2006). Phylogenetic comparisons reveal multiple acquisitions of the toxin genes by enterotoxigenic Escherichia coli strains of different evolutionary lineages. J Clin Microbiol 44, 4528-4536.

Wai, S. N., Takade, A. \& Amako, K. (1995). The release of outer membrane vesicles from the strains of enterotoxigenic Escherichia coli. Microbiol Immunol 39, 451-456.

Warrens, A. N., Jones, M. D. \& Lechler, R. I. (1997). Splicing by overlap extension by PCR using asymmetric amplification: an improved technique for the generation of hybrid proteins of immunological interest. Gene 186, 29-35.

Wright, K. J., Seed, P. C. \& Hultgren, S. J. (2005). Uropathogenic Escherichia coli flagella aid in efficient urinary tract colonization. Infect Immun 73, 7657-7668.

Zhuang, X., Xu, Y., Chong, K., Lan, L., Xue, Y. \& Xu, Z. (2005). OsAGAP, an ARF-GAP from rice, regulates root development mediated by auxin in Arabidopsis. Plant Cell Environ 28, 147-156.

Edited by: I. R. Henderson 